\title{
RESEARCH HIGHLIGHT DH44 neurons: gut-brain amino acid sensors
}

\author{
Yu-Chieh David Chen ${ }^{1}$ and Anupama Dahanukar ${ }^{1,2}$ \\ Cell Research (2018) 28:1048-1049; https://doi.org/10.1038/s41422-018-0101-z
}

\begin{abstract}
Maintaining protein homeostasis is essential for the surviva of animals. A recent study in Cell Research identifies a group of $\mathrm{DH}_{4}{ }^{+}$neuroendocrine cells in the Drosophila brain as a novel fast-acting amino acid sensor, which detects three specific dietary amino acids and promotes food intake.
\end{abstract}

Protein is a major dietary nutrient required for sustaining animal life. Amino acids, building blocks of proteins, are essential for many physiological processes. Selecting and balancing the quality and quantity of food to meet specific metabolic needs relies on multiple sensors in both peripheral and central nervous systems. In the common vinegar fly, Drosophila melanogaster, a number of amino acid detection mechanisms are thought to operate in different life stages. In larvae, at least two amino acid-sensing mechanisms have been reported. First, some dopaminergic neurons in the larval brain act as sensors of essential amino acids via a conserved signal-transducing kinase GCN2 and serve to reject foods that are deficient in essential amino acids. ${ }^{1}$ Second, larval insulin-producing cells (IPCs) can directly sense L-leucine via a leucine transporter, Minidiscs (MND), and regulate release of Drosophila insulin-like peptides (Dilps). ${ }^{2}$ In adult flies, long-term protein deprivation has been shown to induce changes in dopaminergic neuronal circuits and promote protein consumption, ${ }^{3}$ whereas consumption of protein food has been shown to induce the release of Dilp2 via a sexually dimorphic fat body peptide named female-specific independent of transformer (FIT) and suppress further protein intake. ${ }^{4}$ Other than these central mechanisms, peripheral sensing of amino acid and protein requires an ionotropic receptor, IR76b, to drive protein preference and intake. 5,6

A recent article published in Cell Research identifies a group of neuroendocrine cells expressing diuretic hormone 44 (DH44), a homologue of the mammalian corticotropin-releasing hormone $(\mathrm{CRH})$, which directly sense dietary amino acids and promote food consumption. ${ }^{7}$ Using several quantitative feeding assays, the authors show that the presence of three specific amino acids (L-glutamate, L-alanine, and L-aspartate) rapidly stimulates ingestion. Unlike what has been observed previously in feeding choice assays, ${ }^{6}$ the amino acid-stimulated increase in consumption is independent of sex, mating status and internal nutritional state, and thus exposes another aspect of protein homeostasis regulation. Through a targeted genetic RNAi screen designed to identify signaling components of this amino acid-sensing pathway, the authors find that knockdown of either one of the two DH44 receptors (DH44-R1 and -R2) or the DH44 neuropeptide reduces amino acid-stimulated food intake. Mutants lacking $\mathrm{DH} 44$ or its receptor exhibit the same phenotype, suggesting that $\mathrm{DH} 44$ signaling is indeed involved in sensing dietary amino acids. Genetic manipulations of $\mathrm{DH} 44$ neuron activity further strengthen this idea.
To understand whether $\mathrm{DH} 44$ neurons can directly sense amino acids, or whether they are indirectly activated via peripheral taste inputs, the authors combine in vivo and ex vivo calcium imaging analyses of $\mathrm{DH} 44$ neuron responses. Three lines of evidence support a direct role of $\mathrm{DH} 44$ neurons in sensing dietary amino acids. First, $\mathrm{DH} 44$ neurons exhibit concentration-dependent responses to three specific amino acids. Second, responses to these amino acids are observed even if synaptic transmission is blocked by tetrodotoxin. Third, loss of IR76b function, which is required for amino acid responses in peripheral taste neurons, ${ }^{6}$ does not abolish amino acid-stimulated increases in food intake.

The questions that follow are: where and how do $\mathrm{DH} 44$ neurons sense dietary amino acids? Axonal projections of $\mathrm{DH} 44$ neurons have been described to innervate the crop and anterior midgut, ${ }^{8}$ providing potential locations where amino acids may come in contact with neurites of $\mathrm{DH} 44$ neurons in the anterior region of the gastrointestinal tract. Indeed, single-cell RNAseq of DH44 neurons identifies expression enrichment of several putative amino acid transporters, and knocking down two of them (CG13248 and CG4991) abolishes the increase in food intake caused by dietary amino acids. In addition, labeling with a specific antibody against CG13248 shows its expression not only in cell bodies of all six $\mathrm{DH} 44$ neurons but also in their axon terminals in the crop. The mechanisms by which activity in $\mathrm{DH} 44$ axon terminals is back-propagated to the soma remain to be elucidated.

$\mathrm{DH} 44$ neurons have previously been shown to act as nutrient sensors for sugars such as glucose. ${ }^{8}$ The findings of Yang et al. suggest that $\mathrm{DH} 44$ neurons might in fact integrate information about both sugars and amino acids for balancing nutrient intake with metabolic needs. However, the extent of functional overlap in sensing sugars and amino acids is not clear. In fact, Yang et al. find major differences in the downstream cellular signaling events and the properties of stimulus-evoked calcium responses (Fig. 1). DH44 neurons have higher sensitivity to and exhibit faster kinetics in response to amino acids as compared to sugars. In addition, sugars induced calcium oscillation in $\mathrm{DH} 44$ neurons, ${ }^{8}$ whereas Yang et al. observe either calcium oscillations or tonic calcium responses upon amino acid stimulation, indicating the possibility of functional heterogeneity among $\mathrm{DH} 44$ neurons. Interestingly, of the six DH44 neurons in the adult, some were found to co-express Drosophila insulin-like peptide 2 (Dilp2), a known regulator for energy metabolism and growth, and these adult-specific IPCs/ DH44 neurons innervate the crop. ${ }^{9}$ It will be of interest to determine whether Dilp2 expression maps to amino acid-sensing DH44 neurons, positing that as one mechanism by which Dilp release can be regulated under different physiological conditions. Lastly, there is an intriguing possibility of other amino acidsensing neurons in the pars intercerebralis $(\mathrm{PI})$ region, given that

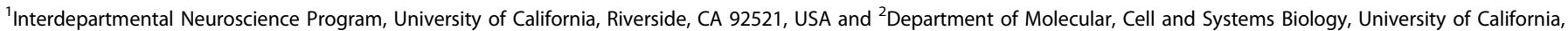
Riverside, CA 92521, USA

Correspondence: Anupama Dahanukar (anupama.dahanukar@ucr.edu) 

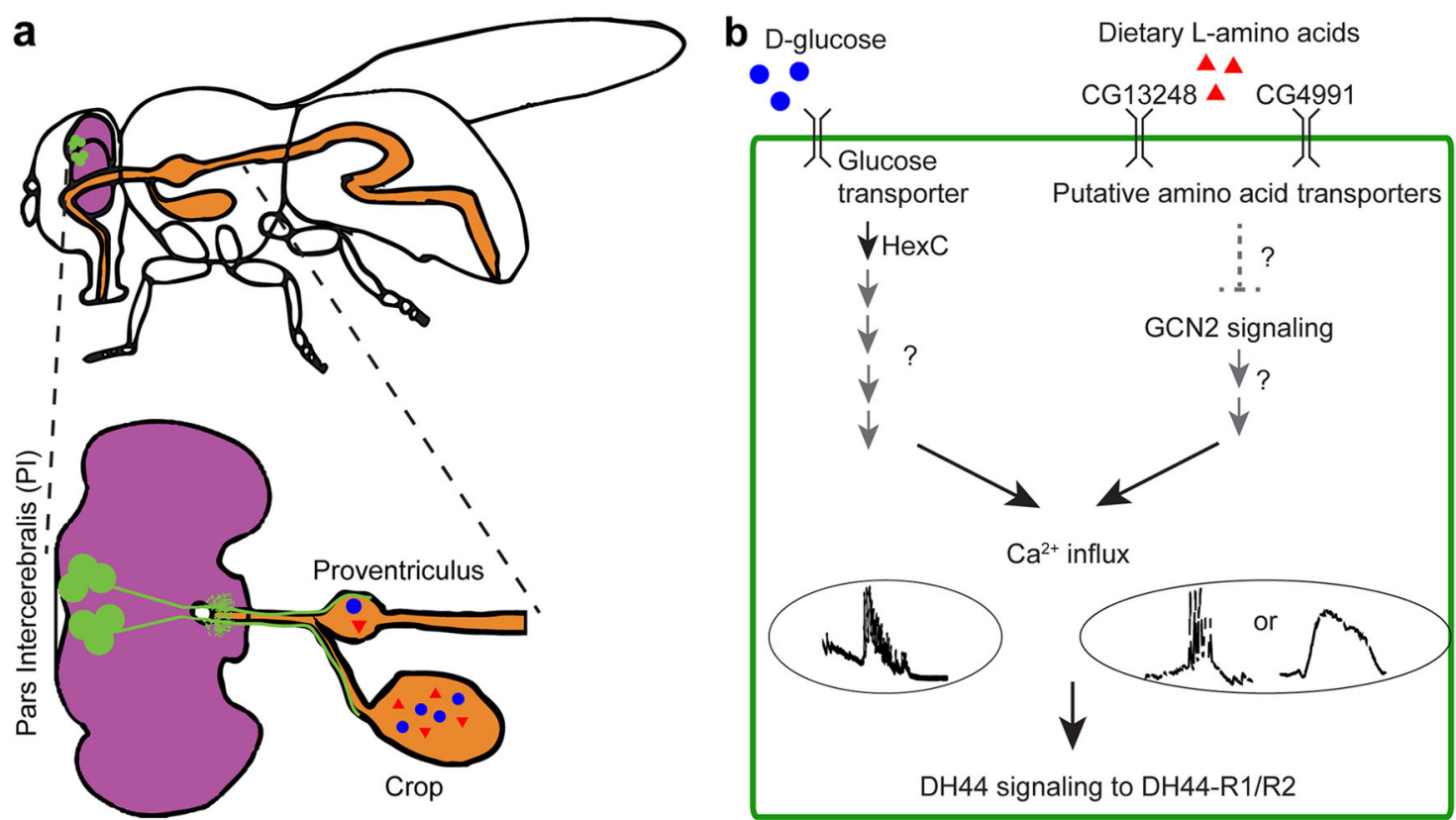

Fig. 1 a Schematic diagram showing innervation patterns of six DH44 neurons (green) in the adult brain (magenta) and gastrointestinal tract (orange). b Cellular signaling events occurring in DH44 neurons in response to sugar and amino acid stimuli

additional neurons juxtaposed with the six $\mathrm{DH} 44$ neurons express the putative amino acid transporter, CG13248. Could these neurons respond to amino acids, and if so, what roles (positive or negative) might they play in controlling protein intake?

The findings of Yang et al. pose a number of interesting questions: Are there other nutrients that activate $\mathrm{DH} 44$ neurons? How do these circuits interact with other known nutrient-sensing circuits? How do DH44-R1 neurons in the central nervous system ${ }^{8}$ act together with DH44-R2 enteroendocrine cells in the gut ${ }^{8}$ to regulate consumption? Since the six $\mathrm{DH} 44$ neurons are preserved through metamorphosis, ${ }^{9}$ do these sensors also function in larvae? What behaviors or physiological processes rely on the small increase in food intake occurring over seconds that is controlled by DH44 signaling? Thus, Yang et al. present a compelling braingut microcircuit that senses three dietary amino acids and promotes intake through $\mathrm{DH} 44$ signaling, along with a starting point for addressing new questions.

\section{ADDITIONAL INFORMATION}

Conflict of interest: The authors declare that they have no conflict of interest.

\section{REFERENCES}

1. Bjordal, M., Arquier, N., Kniazeff, J., Pin, J. P. \& Leopold, P. Cell 156, 510-521 (2014). 2. Maniere, G., Ziegler, A. B., Geillon, F., Featherstone, D. E. \& Grosjean, Y. Cell Rep. 17, 137-148 (2016).

3. Liu, Q. et al. Science 356, 534-539 (2017).

4. Sun, J. et al. Nat. Commun. 8, 14161 (2017).

5. Steck, K. et al. Elife 7, pii: e31625 (2018).

6. Ganguly, A. et al. Cell Rep. 18, 737-750 (2017).

7. Yang, Z. et al. Cell Res. 28, 1013-1025 (2018).

8. Dus, M. et al. Neuron 87, 139-151 (2015).

9. Ohhara, Y., Kobayashi, S., Yamakawa-Kobayashi, K. \& Yamanaka, N. J. Comp. Neurol. 526, 1351-1367 (2018). 